Supporting information for:

\title{
A Method for the Determination of Inorganic Polysulfide Distribution in Aquatic Systems
}

\author{
Alexey Kamyshny, Jr., Irina Ekeltchik, Jenny Gun and Ovadia Lev*
}

\begin{abstract}
The Laboratory of Environmental Chemistry, The Casali Institute of Applied Chemistry, The Chemistry Institute, The Hebrew University of Jerusalem, Jerusalem 91904, Israel. Email: ovadia@vms.huji.ac.il
\end{abstract}

The electronic supporting information contains 5 tables and 1 figure. The first table describes the extraction efficiency of the individual polysulfides vs. the number of extractions (S-1). Three tables (S-2 - S-4) detail the analysis of spiked polysulfides in surface water samples, showing the importance of buffer addition, and provide a comparison to synthetic sample of the same pH. Table S-5 details of the analysis of spiked seawater. Figure S-1 shows the recovery of different polysulfides as a function of added $\mathrm{K}_{2} \mathrm{~S}_{5}$ with and without buffer addition. 
Table S-1. Number of extractions needed to achieve $>94 \%$ recovery (and $>99 \%$ recovery) od synthetic samples spiked with polysulfides.

\begin{tabular}{|c|c|c|c|c|c|c|c|c|c|c|c|c|}
\hline Polysulfide & \multicolumn{4}{|c|}{$\mathrm{Me}_{2} \mathrm{~S}_{3}$} & \multicolumn{4}{|c|}{$\mathrm{Me}_{2} \mathrm{~S}_{4}$} & \multicolumn{4}{|c|}{$\mathrm{Me}_{2} \mathrm{~S}_{5}$} \\
\hline $\begin{array}{l}\text { Extraction } \\
\text { volume, } \mathrm{ml}\end{array}$ & 0.5 & 1 & 2 & 4 & 0.5 & 1 & 2 & 4 & 0.5 & 1 & 2 & 4 \\
\hline $\begin{array}{l}\text { Number of } \\
\text { extractions } \\
\text { for } 94 \% \\
\text { DMPS } \\
\text { recovery } \\
\text { and } 99 \% \\
\text { DMPS } \\
\text { recovery (in } \\
\text { parentheses) }\end{array}$ & $2(2)$ & $2(2)$ & $2(2)$ & 1(1) & $3(3)$ & $2(3)$ & 2(3) & 2(3) & $3(3)$ & $2(3)$ & 2(3) & 1(2) \\
\hline
\end{tabular}

\section{Continued}

\begin{tabular}{|l|l|l|l|l|l|l|l|l|l|l|l|l|}
\hline Polysulfide & \multicolumn{5}{|c|}{$\mathrm{Me}_{2} \mathrm{~S}_{6}$} & \multicolumn{5}{c|}{$\mathrm{Me}_{2} \mathrm{~S}_{7}$} & \multicolumn{3}{|c|}{$\mathrm{Me}_{2} \mathrm{~S}_{8}$} \\
\hline $\begin{array}{l}\text { Extraction } \\
\text { volume, ml }\end{array}$ & 0.5 & 1 & 2 & 4 & 0.5 & 1 & 2 & 4 & 0.5 & 1 & 2 & 4 \\
\hline $\begin{array}{l}\text { Number of } \\
\text { extractions } \\
\text { for 94\% } \\
\begin{array}{l}\text { DMPS } \\
\text { recovery } \\
\text { and 99\% } \\
\text { DMPS } \\
\text { recovery (in } \\
\text { parentheses) }\end{array}\end{array}$ & $2(3)$ & $2(3)$ & $1(2)$ & $1(2)$ & $2(2)$ & $2(2)$ & $1(2)$ & $1(1)$ & $2(2)$ & $2(2)$ & $1(1)$ & $1(1)$ \\
\hline
\end{tabular}


Table S-2 - Analysis of $\mathrm{K}_{2} \mathrm{~S}_{5}$ spiked Lake Kinneret water by protocol 1 without added buffer.

\begin{tabular}{|c|c|c|c|c|c|c|c|c|c|}
\hline $\begin{array}{l}\text { Total } \mathrm{S}^{2-} \text {, } \\
\mathrm{mM}\end{array}$ & $\mathrm{pH}$ & $\begin{array}{l}\text { Ionic } \\
\text { Strength, } \\
\mathrm{mM}\end{array}$ & $\begin{array}{c}\text { Detected } \\
{\left[\mathrm{S}_{3}^{2-}\right](\mu \mathrm{M})} \\
\text { and its } \\
\text { recovery, \% } \\
\text { (in } \\
\text { parentheses) }\end{array}$ & $\begin{array}{c}\text { Detected } \\
{\left[\mathrm{S}_{4}{ }^{2-}\right](\mu \mathrm{M})} \\
\text { and its } \\
\text { recovery, \% } \\
\text { (in } \\
\text { parentheses) }\end{array}$ & $\begin{array}{c}\text { Detected } \\
{\left[\mathrm{S}_{5}^{2-}\right](\mu \mathrm{M})} \\
\text { and its } \\
\text { recovery, \% } \\
\text { (in } \\
\text { parentheses) }\end{array}$ & $\begin{array}{c}\text { Detected } \\
{\left[\mathrm{S}_{6}{ }^{2-}\right](\mu \mathrm{M})} \\
\text { and its } \\
\text { recovery, \% } \\
\text { (in } \\
\text { parentheses) }\end{array}$ & $\begin{array}{c}\text { Detected } \\
{\left[\mathrm{S}_{7}^{2-}\right](\mu \mathrm{M})} \\
\text { and its } \\
\text { recovery, \% } \\
\text { (in } \\
\text { parentheses) }\end{array}$ & $\begin{array}{c}\text { Detected } \\
{\left[\mathrm{S}_{8}{ }^{2-}\right](\mu \mathrm{M})} \\
\text { and its } \\
\text { recovery, \% } \\
\text { (in } \\
\text { parentheses) }\end{array}$ & $\begin{array}{c}\text { Detected } \\
\text { sum }\left[\mathrm{S}_{3}{ }^{2-}\right]- \\
{\left[\mathrm{S}_{8}{ }^{2-}\right](\mu \mathrm{M})} \\
\text { and its } \\
\text { recovery, \% } \\
\text { (in } \\
\text { parentheses) }\end{array}$ \\
\hline 0.25 & 8.67 & 20 & n.d. & n.d. & n.d. & n.d. & $4(60)$ & n.d. & $4(4)$ \\
\hline 0.5 & 8.65 & 25 & n.d.* & $7(17)$ & $20(28)$ & $23(51)$ & $11(89)$ & n.d. & $62(34)$ \\
\hline 1 & 8.75 & 30 & $13(67)$ & $24(24)$ & $74(44)$ & $74(68)$ & $38(135)$ & $6(69)$ & $229(53)$ \\
\hline 2.5 & 8.77 & 55 & 69 (133) & $180(67)$ & $306(66)$ & $256(85)$ & 89 (113) & $14(62)$ & 914 (77) \\
\hline 5 & 8.85 & 90 & 267 (224) & $621(99)$ & 904 (84) & $533(77)$ & $154(84)$ & $20(39)$ & $2499(91)$ \\
\hline $\begin{array}{c}\text { Mean } \\
\text { recovery, } \\
\% \\
\pm \\
\text { Standard } \\
\text { Deviation }\end{array}$ & --- & --- & $141 \pm 79$ & $52 \pm 38$ & $56 \pm 25$ & $70 \pm 15$ & $96 \pm 29$ & $57 \pm 16$ & $52 \pm 35$ \\
\hline
\end{tabular}

* - lower than reported detection limit; not taken into account in the calculation of mean and standard deviation.

Table S-3 - Analysis of $\mathrm{K}_{2} \mathrm{~S}_{5}$ spiked Lake Kinneret water by protocol 1 with added buffer.

\begin{tabular}{|c|c|c|c|c|c|c|c|c|c|}
\hline $\begin{array}{l}\text { Total } \mathrm{S}^{2-} \text {, } \\
\mathrm{mM}\end{array}$ & $\mathrm{pH}$ & $\begin{array}{l}\text { Ionic } \\
\text { Strength, } \\
\mathrm{mM}\end{array}$ & $\begin{array}{c}\text { Detected } \\
{\left[\mathrm{S}_{3}{ }^{2-}\right](\mu \mathrm{M})} \\
\text { and its } \\
\text { recovery, \% } \\
\text { (in } \\
\text { parentheses) }\end{array}$ & $\begin{array}{c}\text { Detected } \\
{\left[\mathrm{S}_{4}{ }^{2-}\right](\mu \mathrm{M})} \\
\text { and its } \\
\text { recovery, \% } \\
\text { (in } \\
\text { parentheses) }\end{array}$ & $\begin{array}{c}\text { Detected } \\
{\left[\mathrm{S}_{5}{ }^{2-}\right](\mu \mathrm{M})} \\
\text { and its } \\
\text { recovery, \% } \\
\text { (in } \\
\text { parentheses) }\end{array}$ & $\begin{array}{c}\text { Detected } \\
{\left[\mathrm{S}_{6}^{2-}\right](\mu \mathrm{M})} \\
\text { and its } \\
\text { recovery, \% } \\
\text { (in } \\
\text { parentheses) }\end{array}$ & $\begin{array}{c}\text { Detected } \\
{\left[\mathrm{S}_{7}^{2-}\right](\mu \mathrm{M})} \\
\text { and its } \\
\text { recovery, \% } \\
\text { (in } \\
\text { parentheses) }\end{array}$ & $\begin{array}{c}\text { Detected } \\
{\left[\mathrm{S}_{8}{ }^{2-}\right](\mu \mathrm{M})} \\
\text { and its } \\
\text { recovery, \% } \\
\text { (in } \\
\text { parentheses) }\end{array}$ & $\begin{array}{c}\text { Detected } \\
\text { sum }\left[\mathrm{S}_{3}{ }^{2-}\right]- \\
{\left[\mathrm{S}_{8}{ }^{-2}\right](\mu \mathrm{M})} \\
\text { and its } \\
\text { recovery, \% } \\
\text { (in } \\
\text { parentheses) }\end{array}$ \\
\hline 0.25 & 8.72 & 100 & n.d. & $26(96)$ & $43(91)$ & $26(85)$ & $14(170)$ & n.d. & 109 (90) \\
\hline 0.5 & 8.69 & 105 & n.d.* & $40(75)$ & $85(93)$ & $72(122)$ & $33(216)$ & n.d. & $230(98)$ \\
\hline 1 & 8.80 & 110 & $26(111)$ & $79(65)$ & $162(78)$ & $129(95)$ & 46 (131) & $6(58)$ & $448(84)$ \\
\hline 2.5 & 8.82 & 135 & 88 (147) & $223(70)$ & $420(77)$ & $285(81)$ & $92(99)$ & $10(40)$ & $1119(80)$ \\
\hline 5 & 8.80 & 170 & 179 (148) & $581(91)$ & $1005(92)$ & $616(87)$ & $161(86)$ & $18(35)$ & $2560(91)$ \\
\hline $\begin{array}{c}\text { Mean } \\
\text { recovery, } \\
\% \\
\pm \\
\text { Standard } \\
\text { Deviation }\end{array}$ & --- & --- & $135 \pm 21$ & $79 \pm 13$ & $86 \pm 8$ & $94 \pm 16$ & $140 \pm 53$ & $44 \pm 12$ & $89 \pm 7$ \\
\hline
\end{tabular}

* - lower than reported detection limit; not taken into account in the calculation of mean and standard deviation. 
Table S-4 - Analysis of $\mathrm{K}_{2} \mathrm{~S}_{5}$ spiked synthetic water held at the $\mathrm{pH}$ of Lake Kinneret water by protocol 1 .

\begin{tabular}{|c|c|c|c|c|c|c|c|c|c|}
\hline $\begin{array}{l}\text { Total } \mathrm{S}^{2-} \text {, } \\
\mathrm{mM}\end{array}$ & $\mathrm{pH}$ & $\begin{array}{l}\text { Ionic } \\
\text { Strength, } \\
\mathrm{mM}\end{array}$ & $\begin{array}{c}\text { Detected } \\
{\left[\mathrm{S}_{3}{ }^{2-}\right](\mu \mathrm{M})} \\
\text { and its } \\
\text { recovery, \% } \\
\text { (in } \\
\text { parentheses) }\end{array}$ & $\begin{array}{c}\text { Detected } \\
{\left[\mathrm{S}_{4}{ }^{2}\right](\mu \mathrm{M})} \\
\text { and its } \\
\text { recovery, \% } \\
\text { (in } \\
\text { parentheses) }\end{array}$ & $\begin{array}{c}\text { Detected } \\
{\left[\mathrm{S}_{5}{ }^{2-}\right](\mu \mathrm{M})} \\
\text { and its } \\
\text { recovery, \% } \\
\text { (in } \\
\text { parentheses) }\end{array}$ & $\begin{array}{c}\text { Detected } \\
{\left[\mathrm{S}_{6}{ }^{2-}\right](\mu \mathrm{M})} \\
\text { and its } \\
\text { recovery, \% } \\
\text { (in } \\
\text { parentheses) }\end{array}$ & $\begin{array}{c}\text { Detected } \\
{\left[\mathrm{S}_{7}^{2-}\right](\mu \mathrm{M})} \\
\text { and its } \\
\text { recovery, \% } \\
\text { (in } \\
\text { parentheses) }\end{array}$ & $\begin{array}{c}\text { Detected } \\
{\left[\mathrm{S}_{8}^{2-}\right](\mu \mathrm{M})} \\
\text { and its } \\
\text { recovery, \% } \\
\text { (in } \\
\text { parentheses) }\end{array}$ & $\begin{array}{c}\text { Detected } \\
\text { sum }\left[\mathrm{S}_{3}{ }^{2-}\right]- \\
{\left[\mathrm{S}_{8}{ }^{-2}\right](\mu \mathrm{M})} \\
\text { and its } \\
\text { recovery, \% } \\
\text { (in } \\
\text { parentheses) }\end{array}$ \\
\hline 0.25 & 8.75 & 150 & n.d. * & $23(76)$ & $58(114)$ & $40(122)$ & $16(189)$ & n.d. & $138(105)$ \\
\hline 0.5 & 8.64 & 150 & $18(176)$ & $43(83)$ & $72(80)$ & $64(110)$ & $35(230)$ & $3(75)$ & $236(102)$ \\
\hline 1 & 8.75 & 150 & $39(172)$ & $89(75)$ & $163(79)$ & $125(95)$ & 48 (137) & $7(74)$ & $471(90)$ \\
\hline 2.5 & 8.74 & 150 & 78 (139) & $288(98)$ & 489 (96) & $289(89)$ & $85(99)$ & $13(52)$ & $1242(96)$ \\
\hline 5 & 8.84 & 155 & 239 (193) & 649 (99) & $910(80)$ & $460(63)$ & $132(69)$ & $14(26)$ & 2404 (83) \\
\hline 10 & 8.83 & 160 & $698(284)$ & 1471 (113) & $1583(70)$ & 775 (54) & $234(61)$ & $24(22)$ & $4785(83)$ \\
\hline $\begin{array}{c}\text { Mean } \\
\text { recovery, } \\
\% \\
\pm \\
\text { Standard } \\
\text { Deviation }\end{array}$ & --- & --- & $193 \pm 55$ & $91 \pm 15$ & $87 \pm 16$ & $89 \pm 26$ & $131 \pm 68$ & $50 \pm 25$ & $93 \pm 9$ \\
\hline
\end{tabular}

* - lower than reported detection limit; not taken into account in the calculation of mean and standard deviation.

Table S-5 - Analysis of $\mathrm{K}_{2} \mathrm{~S}_{5}$ spiked seawater by protocol 1. $\mathrm{pH}=8.09$ in all samples.

\begin{tabular}{|c|c|c|c|c|c|}
\hline $\begin{array}{c}\text { Total } \mathrm{S}^{2-}, \\
\mu \mathrm{M}\end{array}$ & $\begin{array}{c}\text { Detected } \\
{\left[\mathrm{S}_{4}{ }^{2-}\right](\mu \mathrm{M})} \\
\text { and its } \\
\text { recovery, } \% \\
\text { (in } \\
\text { parentheses) }\end{array}$ & $\begin{array}{c}\text { Detected } \\
{\left[\mathrm{S}_{5}{ }^{2-}\right](\mu \mathrm{M})} \\
\text { and its } \\
\text { recovery, \% } \\
\text { (in } \\
\text { parentheses) }\end{array}$ & $\begin{array}{c}\text { Detected } \\
{\left[\mathrm{S}_{6}{ }^{2-}\right](\mu \mathrm{M})} \\
\text { and its } \\
\text { recovery, \% } \\
\text { (in } \\
\text { parentheses) }\end{array}$ & $\begin{array}{c}\text { Detected } \\
{\left[\mathrm{S}_{7}{ }^{2-}\right](\mu \mathrm{M})} \\
\text { and its } \\
\text { recovery, } \% \\
\text { (in } \\
\text { parentheses) }\end{array}$ & $\begin{array}{c}\text { Detected } \\
\text { sum }\left[\mathrm{S}_{4}{ }^{2-}\right]- \\
{\left[\mathrm{S}_{7}{ }^{2-}\right](\mu \mathrm{M})} \\
\text { and its } \\
\text { recovery, } \% \\
\text { in } \\
\text { parentheses) }\end{array}$ \\
\hline 3000 & $198(127)$ & $222(83)$ & $174(102)$ & $28.9(64)$ & $622(98)$ \\
\hline 500 & $21.7(83)$ & $32.1(72)$ & $21.2(84)$ & $5.25(70)$ & $80.2(75)$ \\
\hline 250 & $17.2(132)$ & $17.2(78)$ & $10.1(71)$ & $3.49(93)$ & $48.0(90)$ \\
\hline 100 & $5.62(108)$ & $8.01(90)$ & $4.59(81)$ & $2.00(134)$ & $20.2(95)$ \\
\hline $\begin{array}{c}\text { Mean } \\
\text { recovery, \% } \\
\pm \text { Standard } \\
\text { Deviation }\end{array}$ & $113 \pm 22$ & $81 \pm 8$ & $82 \pm 14$ & $90 \pm 31$ & $90 \pm 10$ \\
\hline
\end{tabular}


Figure S-1. a) Recovery of the total polysulfide as a function of concentration of dissolved polysulfide precursor $\left(\mathrm{K}_{2} \mathrm{~S}_{5}\right)$ in the spiking experiments for spiked distilled water spiked (closed circle); Lake Kinnret water spiked with $\mathrm{K}_{2} \mathrm{~S}_{5}$ (closed rectangle); Lake Kinnret water spiked with $\mathrm{K}_{2} \mathrm{~S}_{5}$ with buffer added to the derivatization mixture before methylation (closed triangle). b) Reconstruction of the data of frame A with xaxis denoting the calculated ionic strength.
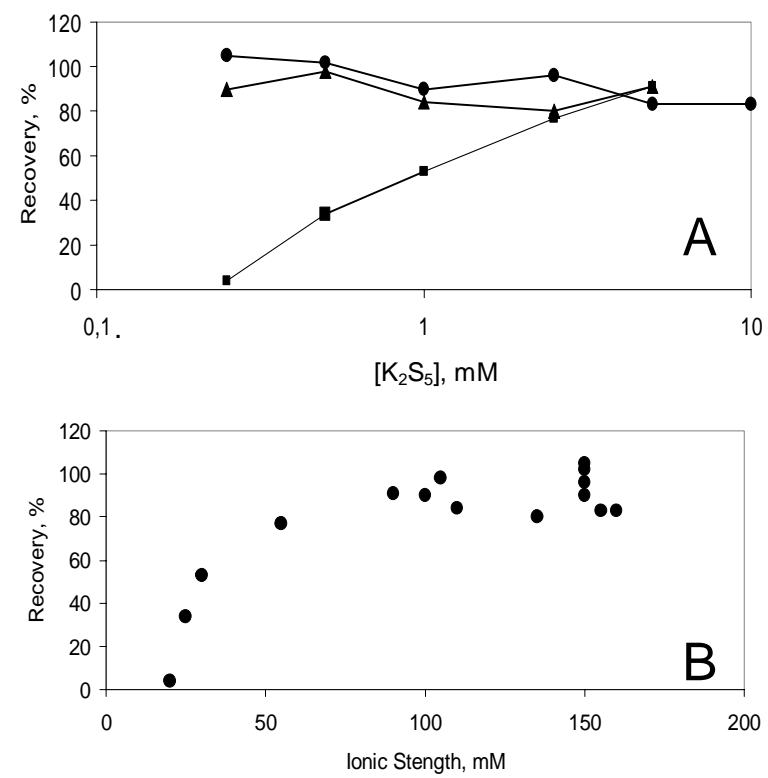